\title{
Medical Management of Symptomatic Fibroids: Worth It?
}

\author{
Chandrashekhar V. Hegde ${ }^{1}$
}

Received: 26 May 2017/Accepted: 29 May 2017/Published online: 10 June 2017

(C) Federation of Obstetric \& Gynecological Societies of India 2017

\begin{abstract}
About the Author

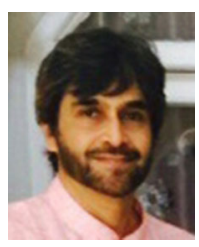

Dr. Chandrashekhar V. Hegde is Editor in chief of "The Journal of Obstetrics and Gynecology of India." He is also Professor and Head of Department at the T N Medical College and BYL Nair Hospital, Mumbai. He is also Visiting Consultant at the Sir HN Reliance Foundation Hospital, Mumbai. He has a rich teaching experience of more than three decades and has taught and trained a large number of undergraduate and postgraduate students in that duration of time. He has been a postgraduate and undergraduate examiner in Obstetrics and Gynecology at the Bombay University and the Maharashtra University of Health Sciences. He runs the PICSEP program of the Journal and FOGSI under whose aegis, and ten programs in research methodology are conducted all over the country every year. This program aims at improving the quality of original research papers being submitted to the journal. He has several research papers published in this journal and several other journals of standing. He is the winner of the prestigious FOGSI Corion award in the senior category. He has more than 50 videos on the YouTube on all aspects of vaginal and endoscopic surgery, solely for the purpose of furthering medical education. His other interests include nonfiction reading and reading blogs on Indian history and contemporary politics.
\end{abstract}

The problems of anemia, pain/dysmenorrhea, menorrhagia and infertility posed by fibroids continue to be treated largely by a combination of surgery (myomectomy/hysterectomy), medication (gonadotropin-releasing hormone agonists $\{\mathrm{GnRH}\}$, progestogens, etc.), magnetic resonanceguided focused ultrasound surgery and uterine artery embolization among other techniques [1-4].

Medical treatment in isolation for fibroids or as a preparatory for surgery includes nonsteroidal anti-

Dr. Chandrashekhar V. Hegde is Professor and Head of the Department of Obstetrics and Gynaecology in T N Medical College and B Y L Nair Hospital Mumbai 400008.

Chandrashekhar V. Hegde

dr.c.v.hegde@gmail.com

1 Mumbai, India inflammatory drugs, oral contraceptives, intrauterine devices and iron supplements. The use of oral progestin is limited, because it may cause breakthrough bleeding and promote the proliferation of cells in the myoma $[5,6]$. Moreover, the effects of progestin as a treatment for symptoms associated with fibroids have been poorly evaluated. The only definitive medical therapy was $\mathrm{GnRH}$ (with all its known side effects) till recently.

There could be a large subset of women who would prefer medical treatment to surgery or use medical treatment as an adjunct to surgery or in whom surgery may be contraindicated or are in situations where surgery could affect fertility adversely and that is where Ullipristal Acetate (UPA) steps right in. UPA is a progesterone receptor modulator, a synthetic steroid derived from 19-norprogesterone, and shows tissue-specific agonist, antagonist or combined activity in target cells [7-9]. It also 
modulates the expression of vascular endothelial growth factors and hormone receptors and modulates extracellular matrix breakdown in leiomyoma cells but not in myometrial cells. Since it blocks progesterone receptors, it reduces the positive effect of progesterone on the growth of fibroids and helps in reducing its size. Therefore, presumably it should have a positive role in reducing bleeding and anemia due to fibroids. The half-life of UPA is about 38 hours with most of the metabolites being excreted in the feces. Medications like carbamazepine, phenytoin and erythromycin which affect the metabolism of UPA have to be taken with care. GnRH analogs too are used in the management of symptomatic fibroids. GnRH analogs in an offlabel use were prescribed in a Canadian study [10] to reduce anemia and fibroid size. GnRH agonists reduce bleeding, anemia, pain and the volume of fibroids [11]. However, these effects are transient, and the fibroids usually return to pre-therapy size within a few months of discontinuation [12]. The median rate of fibroid volume reduction with $\mathrm{GnRH}$ agonist therapy reportedly ranges from 42 to $58.3 \%$ [13, 14]. In a Korean study [15], two groups of patients with symptomatic fibroids were treated with UPA and GnRH analog leuprolide acetate. In total, 51 patients were treated with $5 \mathrm{mg}$ UPA administered orally for 3 months and the 50 patients in the GnRH agonist group received subcutaneous injections of $3.75 \mathrm{mg}$ leuprolide acetate once a month for 3 months. The median largest diameter of leiomyoma in pre-treatment was $8.1 \mathrm{~cm}$ in the UPA group and $9.0 \mathrm{~cm}$ in the GnRH agonist group $(P=0.165)$. The median volume of three largest leiomyomas was $233.6 \mathrm{~cm}^{3}$ in the UPA group and $289.0 \mathrm{~cm}^{3}$ (in the GnRH agonist group $(P=0.435)$, and the types of leiomyomas did not differ significantly between the groups. In this study, median fibroid volume reduction was more in the GnRH group than the UPA group.

Four trials (PEARL I, II, III and IV) involved the usage of UPA. Treatment began in the first week of the menstrual period. PEARL I involved an attempt to note the effect of UPA on anemia due to heavy periods because of fibroids and where there were plans to have a surgical intervention. PEARL I showed that a higher dose $(10 \mathrm{mg})$ of UPA reduced bleeding completely (amenorrhea) as compared to a lesser dose $(5 \mathrm{mg})$. Overall, bleeding was reduced by $90 \%$ of users of UPA. However, the median total fibroid volume reduced by a lesser extent in those who used a 10-mg dose daily as compared to a 5-mg dose. This was after a trial period of 13 weeks. No fibroid was more than $10 \mathrm{~cm}$ in this trial [16].

PEARL II enrolled those who had heavy bleeding and were eligible for surgical treatment. Here, daily UPA was compared with monthly injections of leuprolein a GnRH agonist. It was seen that after 13 weeks of treatment a dose of $10 \mathrm{mg}$ UPA controlled bleeding in $98 \%$ of patients as opposed to $90 \%$ in those who used $5 \mathrm{mg}$ UPA per day. Leuprolein too did not fare badly; bleeding was controlled in $89 \%$ of users. However, leuprolein had the best effect in reducing median fibroid size as compared to UPA [13]. Hot flushes were much less in the UPA group than the leuprolein group. From the PEARL I and II trials, the dose of $5 \mathrm{mg}$ per day was sought to be defined as the minimum dose of UPA.

In PEARL III, the primary outcome was amenorrhea. UPA was started within the first 4 days of menstruation. "Patients with heavy bleeding and at least one fibroid took open-label ulipristal $10 \mathrm{mg}$ for 3 months. This was followed by double-blind treatment with norethisterone or a placebo for 10 days. The women could then opt to repeat this regimen up to three times giving a total of up to four courses". After the first course of UPA, each woman could choose either to leave the study and attend a final follow-up visit at week 12, or to be assessed for a further 18 months and enroll in the PEARL III extension study to obtain three further 3-month long courses, with each course of UPA treatment separated by two menstrual cycles (approximately 2 months). In total, $79 \%$ of women achieved amenorrhea after one course of treatment of 13 weeks of UPA. In those who opted for four courses of treatment, $90 \%$ had amenorrhea. With every additional course, the amenorrhea rates increased. Fibroids shrunk more in those who had a four course of treatment of UPA as compared to those who had one course of treatment [17].

PEARL IV also had amenorrhea as a primary endpoint. Patients were randomised to take UPA 5 or $10 \mathrm{mg}$ in 12-week courses. After four treatment courses, amenorrhea was seen in $63 \%$ of those who took $5 \mathrm{mg}$ UPA per day as compared to $73 \%$ of those who took $10 \mathrm{mg}$ UPA per day. Fibroid volume reduced by $73 \%$ in both groups [18].

From studying the outcome of the four PEARL trials on various endpoints, the effect of UPA seems more potent with a dose of $10 \mathrm{mg}$ rather than $5 \mathrm{mg}$ over four courses of 3 months each. Amenorrhea, reduced bleeding, median decrease in the size of fibroids were variables which were achieved with a higher dose of UPA, though a GnRH agonist leuprolein had a greater effect in reducing the median size of fibroids as compared to UPA. According to Szamatowicz and Kotarski [19], fibroid volume may stay reduced for up to 6 months after completion of treatment with UPA. Additionally, the quality of life could improve and ovulation and menstruation could resume soon after stopping treatment.

The adverse effects of UPA seen in the PEARL trials included nausea, headache, abdominal pain, discomfort, breast tenderness and hot flushes.

The use of GnRH though in the pre-treatment of fibroids prior to surgery has been well established. The effects are 
temporary, and side effects like bone mineral density loss and cardiovascular side effects limit its use [20].

The effects of UPA on the endometrium needed to be studied only because the endometrium is vulnerable to the unopposed action of estrogen given that UPA is progesterone blocker. The real dangers of endometrial hyperplasia and endometrioid carcinoma were a threat. In a workshop in Bethesda, experts studied the interpretations of endometrial samples of patients on UPA and did not find premalignant or malignant changes. Most endometrial patterns were benign or resembled that seen in a normal endometrial cycle. However, some changes were unique and were termed progesterone receptor modulators-associated endometrial changes (PAECs) [21]. An annual ultrasound could be recommended in patients on prolonged therapy and endometrial biopsies obtained when deemed necessary.

Though the therapeutic daily dose of UPA ( $5 \mathrm{mg}$ ) may result in suppression of ovulation, nonhormonal contraception is advised to prevent an unwanted pregnancy. Menstruation usually returns within 1 month of stoppage of UPA. Pregnancy and lactation are contraindications for usage of UPA. Luyckx et al. [22] reported the first series of pregnancies achieved after UPA treatment for uterine fibroids. There were no fibroid-related complications during pregnancy and no fibroid growth in pregnancy probably due to apoptosis as per this study.

Any healthcare system has to reckon with cost considerations of treatment protocols when different molecules could be used for a similar expected outcome. A study from the Netherlands found that UPA was less expensive as compared to leuprolide and resulted in a saving to the healthcare system [23]. A Canadian study too has mentioned about the cost saving by the usage of UPA to its healthcare system as against the usage of GnRH. The same article underscores the utility of UPA in the quick control of menstrual bleeding, with few side effects and the subsequent improvement in the quality of life [24].

In a multicenter randomised controlled trial, mifepristone in doses of 5 and $10 \mathrm{mg}$ daily was administered to women with symptomatic fibroids. Some women were administered $3.75 \mathrm{mg}$ intramuscular enantone (leuprolein) monthly. Most of the women became amenorrhoeic, and dysmenorrhea and pelvic pain were reduced. The mean volume of the largest fibroid was reduced almost equally in all the groups with minimal adverse effects [25].

GnRH and selective progesterone receptor modulators ultimately seem to be the mainstay of medical treatment of symptomatic fibroids with surgery still being a definitive form of treatment. The changes induced by medical treatment like reduced bleeding/amenorrhea, reduced fibroid volume may be used with advantage selectively when deemed necessary. Reduction in the size and volume of fibroids may make resection less troublesome at hysteroscopy. Likewise, pre-treatment for 3 months by UPA and mifepristone may render laparoscopic myomectomy easier. Adverse events especially if monthly injections of GnRH analogs are used will need to be looked out for. As of now, there is no timeline for postmedical treatment surgical intervention. The changes induced by medical treatment are transient, and status quo is restored a few months after stoppage of treatment.

The search for the holy grail for the medical treatment of fibroids goes on. The moot question, however, still remains largely unanswered-is it worth it?

\section{References}

1. Talaulikar VS, Manyonda IT. Ulipristal acetate: a novel option for the medical management of symptomatic uterine fibroids. Adv Ther. 2012;29:655-63.

2. McCarthy-Keith DM, Armstrong AY. Innovations in uterine fibroid therapy. Therapy. 2011;8:189-200.

3. Tempany CM, Stewart EA, McDannold N, et al. MR imagingguided focused ultrasound surgery of uterine leiomyomas: a feasibility study. Radiology. 2003;226:897-905.

4. Tropeano G, Amoroso S, Scambia G. Non-surgical management of uterine fibroids. Hum Reprod Update. 2008;14:259-74.

5. Nisolle M, Gillerot S, Casanas-Roux F, et al. Immunohistochemical study of the proliferation index, oestrogen receptors and progesterone receptors $\mathrm{A}$ and $\mathrm{B}$ in leiomyomata and normal myometrium during the menstrual cycle and under gonadotrophin-releasing hormone agonist therapy. Hum Reprod. 1999; 14(11):2844-50.

6. Kim JJ, Sefton EC. The role of progesterone signaling in the pathogenesis of uterine leiomyoma. Mol Cell Endocrinol. 2012;358(2):223-31.

7. Chwalisz K, Perez MC, Demanno D, et al. Selective progesterone receptor modulator development and use in the treatment of leiomyomata and endometriosis. Endocr Rev. 2005;26:423-38.

8. Blithe DL, Nieman LK, Blye RP, et al. Development of the selective progesterone receptor modulator CDB-2914 for clinical indications. Steroids. 2003;68:1013-7.

9. Croxtall JD. Ulipristal acetate: in uterine fibroids. Drugs. 2012;72(8):1075-85.

10. Lefebvre G, Vilos G, Allaire C, et al. The management of uterine leiomyomas. J Obstet Gynaecol Can. 2003;25(5):396-418.

11. Lethaby A, Vollenhoven B, Sowter M. Efficacy of pre-operative gonadotrophin hormone releasing analogues for women with uterine fibroids undergoing hysterectomy or myomectomy: a systematic review. BJOG. 2002;109(10):1097-108.

12. Ortmann O, Diedrich K. Pituitary and extrapituitary actions of gonadotrophin-releasing hormone and its analogues. Hum Reprod. 1999;14(Suppl 1):194-206.

13. Donnez J, Tomaszewski J, Vazquez F, et al. Ulipristal acetate versus leuprolide acetate for uterine fibroids. N Engl J Med. 2012;366:421-32. doi:10.1056/NEJMoa1103180.

14. Mavrelos D, Ben-Nagi J, Davies A, et al. The value of preoperative treatment with GnRH analogues in women with submucous fibroids: a double-blind, placebo-controlled randomized trial. Hum Reprod. 2010;25:2264-9.

15. Lee MJ, Yun BS, Seong SJ, et al. Uterine fibroid shrinkage after short-term use of selective progesterone receptor modulator or 
gonadotropin-releasing hormone agonist. Obstet Gynecol Sci. 2017;60(1):69-73.

16. Donnez J, Tatarchuk TF, Bouchard P, et al. Ulipristal acetate versus placebo for fibroid treatment before surgery. N Engl J Med. 2012;366:409-20. doi:10.1056/NEJMoa1103182.

17. Donnez J, Vázquez F, Tomaszewski J, et al. Long-term treatment of uterine fibroids with ulipristal acetate. Fertil Steril. 2014;101:1565.e1-1573.e1. doi:10.1016/j.fertnstert.2014.02.008.

18. Donnez J, Donnez O, Matule D, et al. Long-term medical management of uterine fibroids with ulipristal acetate. Fertil Steril. 2016;105:165.e4-173.e4. doi:10.1016/j.fertnstert.2015.09.032.

19. Szamatowicz M, Kotarski J. Selective progesterone receptor modulator ulipristal acetate-a new option in the pharmacological treatment of uterine fibroids in women. Ginekol Pol. 2013;84(3):219-22.

20. Broekmans FJ. GnRH agonists and uterine leiomyomas. Hum Reprod. 1996;11(Suppl 3):3-25.

21. Williams AR, Bergeron C, Barlow DH, et al. Endometrial morphology after treatment of uterine fibroids with the selective progesterone receptor modulator, ulipristal acetate. Int J Gynecol Pathol. 2012;31(6):556-69.
22. Luyckx M, Squifflet JL, Jadoul P, et al. First series of 18 pregnancies after ulipristal acetate treatment for uterine fibroids. Fertil Steril. 2014;102(5):1404-9.

23. Zakiyah N, van Asselt AD, Postma MJ. Ulipristal acetate for preoperative treatment of moderate-to-severe uterine fibroids in women of reproductive age in The Netherlands: cost minimization analysis and budget impact analysis. J Med Econ. 2017;20(3):280-7. doi:10.1080/13696998.2016.1254090 Epub 2016 Nov 11.

24. Tsoi B, Blackhouse G, Ferrazzi S, et al. Incorporating ulipristal acetate in the care of symptomatic uterine fibroids: a Canadian cost-utility analysis of pharmacotherapy management. Clinicoecon Outcomes Res. 2015;7:213-25. doi: 10.2147/CEOR.S78115.

25. Liu C, Lu Q, Qu H, et al. Different dosages of mifepristone versus enantone to treat uterine fibroids: a multicenter randomized controlled trial. Medicine. 2017;96(7):e6124 (ISSN: 1536-5964). 